\title{
Interface Dynamics in the Transcritical Flow of Liquid Fuels into High-Pressure Combustors
}

\author{
Lluís Jofre* and Javier Urzay ${ }^{\dagger}$ \\ Center for Turbulence Research, Stanford University, Stanford, CA 94305, USA
}

\begin{abstract}
Rocket engines and new generations of high-pressure gas turbines and diesel engines oftentimes involve atomization, vaporization and combustion of propellants injected at subcritical temperature into an environment at a pressure larger than that of the corresponding critical points of the individual components of the mixture. This class of trajectories in the thermodynamic space have been referred to as transcritical. As a result, relatively sharp interfaces may persist in pressure and temperature conditions where they were not expected to exist. This is particularly relevant in hydrocarbon-fueled mixtures that display a critical-point elevation property by which the two-phase region extends up to pressures much larger than the critical pressures of the individual components. As a consequence, linear thermodynamic trajectories emulating typical injection conditions frequently pass through the two-phase region, thus indicating that the mixture may become separated there into liquid and vapor phases by an interface. In this study, a set of modifications to the Navier-Stokes equations for multi-component flows is proposed based on diffuse-interface theory in order to treat the emergent and vanishing interfaces in the same flow field. This requires appropriate alterations of the stress tensor and diffusive fluxes of heat and species. The resulting transport formulation is particularized for binary mixtures, as well as collapsed to single-component gradient theory for stationary quasi-planar vapor-liquid interfaces.
\end{abstract}

\section{Nomenclature}

$a, b \quad$ Equation of state coefficients

$c \quad$ Molar density, $\mathrm{mol} / \mathrm{m}^{3}$

$D \quad$ Diffusion coefficient, $\mathrm{m}^{2} / \mathrm{s}$

$E \quad$ Specific total energy, $\mathrm{J} / \mathrm{kg}$

$e \quad$ Specific internal energy, $\mathrm{J} / \mathrm{kg}$

$F \quad$ Helmholtz free energy, J

$f \quad$ Specific Helmholtz free energy, $\mathrm{J} / \mathrm{kg}$

$G \quad$ Gibbs free energy, J

$h \quad$ Specific enthalpy, $\mathrm{J} / \mathrm{kg}$

$\mathbf{J}, \mathcal{J}$ Species diffusion fluxes, $\mathrm{kg} /\left(\mathrm{s} \cdot \mathrm{m}^{2}\right)$

$N \quad$ Number of species

$n \quad$ Number of mols

$N_{A} \quad$ Avogadro's number, $1 / \mathrm{mol}$

$P$ Pressure, bar

q, $\mathcal{Q}$ Heat diffusion fluxes, $\mathrm{J} /\left(\mathrm{s} \cdot \mathrm{m}^{2}\right)$

$\boldsymbol{q}_{\boldsymbol{c}} \quad$ Heat conduction flux, $\mathrm{J} /\left(\mathrm{s} \cdot \mathrm{m}^{2}\right)$

$R^{0} \quad$ Ideal gas constant, $\mathrm{J} /(\mathrm{K} \cdot \mathrm{mol})$

$s \quad$ Specific entropy, $\mathrm{J} /(\mathrm{kg} \cdot \mathrm{K})$

$\dot{s}_{\text {prod }}$ Entropy production rate, $\mathrm{J} /\left(\mathrm{K} \cdot \mathrm{s} \cdot \mathrm{m}^{3}\right)$

$T$ Temperature, $\mathrm{K}$ $t$ Time, s

$\mathbf{v}$ velocity vector, $\mathrm{m} / \mathrm{s}$

$v \quad$ Molar volume, $\mathrm{mol} / \mathrm{kg}$

$W \quad$ Molecular weight, $\mathrm{kg} / \mathrm{mol}$

$X \quad$ Molar fraction

$Y \quad$ Mass fraction

$Z \quad$ Compressibility factor

$\eta, \zeta \quad$ Shear and bulk viscosities, $\mathrm{Pa} \cdot \mathrm{s}$

$\kappa \quad$ Gradient-energy coefficient, $\mathrm{m}^{7} /\left(\mathrm{kg} \cdot \mathrm{s}^{2}\right)$

$\lambda$ Thermal conductivity, $\mathrm{W} /(\mathrm{m} \cdot \mathrm{K})$

$\bar{\mu} \quad$ Molar chemical potential, $\mathrm{J} / \mathrm{mol}$

$\rho \quad$ Density, $\mathrm{kg} / \mathrm{m}^{3}$

$\sigma \quad$ Surface-tension coefficient, $\mathrm{N} / \mathrm{m}$

$\tau, \mathcal{K}$ Stress tensors, $\mathrm{N} / \mathrm{m}^{2}$

$\varphi \quad$ Fugacity coefficient

Subscripts

$c \quad$ Critical point

$i, j \quad$ Species index

$N L \quad$ Non-local quantity

*Postdoctoral Researcher. E-mail: jofre@stanford.edu

†Senior Research Engineer. E-mail: jurzay@stanford.edu 


\section{Introduction}

The characteristic pressure and temperature of the burnt gases in the combustor of Apollo's Saturn-V F-1 rocket engines were 77.5 bar and $3572 \mathrm{~K}$. The engine operated a mixture of liquid oxygen (critical pressure $P_{c}=50$ bar; critical temperature $\left.T_{c}=155 \mathrm{~K}\right)$ and RP-1 fuel $\left(P_{c}=21\right.$ bar; $\left.T_{c}=662 \mathrm{~K}\right)$. The typical injection temperatures of RP-1 and liquid oxygen were $311 \mathrm{~K}$ and $97 \mathrm{~K}$, respectively. The propellants were injected separately into the combustion chamber through a multi-perforated plate that forced their atomization and mixing by delivering them as mutually impinging jets.

The F-1 rocket engine is a classical example of a contemporary generation of high-power aero-propulsion devices that pushed the operating conditions above the critical points of the propellants. The technical command of such high combustion pressures represented a cornerstone in enabling the large power and specific impulse necessary for a manned mission to the Moon. However, the extreme operating conditions brought along several engineering challenges, among which the most critical one for the Apollo Program proved to be that of combustion instabilities created by the injection configuration. ${ }^{1}$ Early analyses of this problem recognized the conceptual difficulties related to describing atomization, vaporization and combustion of propellants injected at subcritical temperatures into an environment at a pressure larger than that of their corresponding critical points. ${ }^{2}$ This class of trajectories in the thermodynamic space have been referred to as transcritical in more recent literature. ${ }^{3}$ The deleterious combustion pressure waves present in the first designs of the F-1 rocket engines were removed by modifying the injection plate with a number of baffles, since the phenomenon was sensitive to the characteristic distance from the injection plate to the beginning of the combustion zone. Paradoxically, that distance appears to be a quantity hardly possible to predict even nowadays with current theoretical and computational models for reasons explained later in this section.

The study of transcritical dynamics also finds important applications in recent designs of gas turbine engines for jet propulsion. In particular, current trends in ultra-low emission technologies for aviation industry are gearing combustors toward lean burn and high pressure ratios. Lean burn aims at decreasing nitrogen oxides by avoiding their peak production rate at stoichiometry, but requires an intense dilution and mixing of the fuel with the air entering the combustor. Similarly, high pressure ratios are employed to increase engine power and reduce emissions of carbon oxides and unburnt hydrocarbons. These pressure ratios yield combustor pressures of order 45 bar at takeoff, while most jet fuels have critical pressures in the range 15-22 bar. In this way, transcritical conditions likely develop in the combustor that may have an impact on the fuel-air mixing characteristics because of the alteration of the classic spray atomization dynamics expected at lower pressures. However, the extent of these effects remains mostly unknown. Recent experimental observations of similar aspects have been made within the context of diesel engines by Dahms and coworkers. ${ }^{4}$

The challenge for predictive calculations of transcritical phenomena is the complexity of the transitional character of liquid breakup, dispersion and vaporization as conditions approach the critical point. To understand this, consider first the subcritical limit in which the liquid is injected into a hot gas environment whose pressure is lower than the critical pressure of the liquid. In this limit, the liquid-to-gas density ratio is large, and the liquid atomizes following classic dynamics reported in several studies. ${ }^{5}$ The resulting thickness of the liquid-gas interface is clearly not in the continuum range and can be taken to be infinitesimally small in hydrodynamic scales. Additionally, a relatively large amount of energy must be provided by the gas in order to heat up and vaporize the liquid phase. As a result, the beginning of the vaporization stage is delayed farther downstream until the liquid has broken up into a sufficiently dilute cloud of droplets. ${ }^{6}$ However, as the pressure is increased above the critical point of the liquid, the liquid-gas density ratio decreases because of an increase in the density of the gas environment. The interface becomes thicker as the liquid receives heat from the combustor environment and its temperature nears the critical temperature. This is accompanied by a decrease in surface tension and vaporization enthalpy, in a manner that makes the atomization process to increasingly resemble one at infinite Weber numbers followed by rapid mixing with the gas environment without significant energy barrier for vaporization.

Heavy hydrocarbons require increments of temperature of order $300-400 \mathrm{~K}$ in order to reach their critical points. For these fuels, it is conceptually plausible that finite surface-tension and vaporization effects persist longer in the combustor. However, the problem becomes exceedingly complex in mixtures of hydrocarbons and typical oxidizers, in that the resulting phase diagram displays critical-point elevation properties that, depending on the local composition, may lead to locally subcritical conditions even if the pressure is much larger than the corresponding critical values of the separate components. This may lead to the persistence of relatively sharp interfaces in pressure and temperature conditions where they were not expected to exist. 
Note that for single-component systems consisting of a liquid atomizing in its own vapor, the description of the dynamics becomes much simpler, in that there is practically no distinction between the two phases at pressures above the critical point. Accordingly, the interface disappears, and the surface tension vanishes. The single-component case, however, is not the one found in most practical applications. Additional factors that prevent further understanding of transcritical dynamics are the large uncertainties in high-pressure physical properties of complex mixtures of reactants and combustion products, and the lack of quantitative experimental diagnostics for model validation in such extreme environments.

This work addresses basic theoretical aspects of flow transport under transcritical conditions. It is organized as follows. In Section II, a phase diagram for a typical hydrocarbon-fueled system is described that illustrates the thermodynamic space of solutions at high pressures and describes critical-point elevation properties. Section III is devoted to a derivation of a set of conservation equations that simultaneously consider surface-tension effects along with relatively permeable interfaces. Lastly, conclusions are provided in Section IV . This report builds on recent analyses from Ref. ${ }^{7}$ by providing different perspectives of the formulation and additional considerations that may be of practical use in the computation of hydrocarbonfueled transcritical flows.

\section{Phase diagrams and critical-point elevation properties of hydrocarbon-fueled mixtures}

Some insight can be gained into system trajectories leading to transcritical regimes by studying vaporliquid equilibrium curves. These correspond to boundaries of the region within which the system separates into two or more different phases across an interface. The phase diagram illustrates the thermodynamic space of solutions of the problem, but does not provide any information about the dynamics. For instance, the liquid-fuel stream and the hot gas environment may be both represented by two different points in the phase diagram, but the thermodynamic trajectories of the mixture elements are solutions to the conservation equations and the associated boundary conditions. In this section, mixtures of n-dodecane $\left(P_{c, 1}=18\right.$ bar, $\left.T_{c, 1}=658 \mathrm{~K}\right)$ and nitrogen $\left(P_{c, 2}=34 \mathrm{bar}, T_{c, 2}=126 \mathrm{~K}\right)$ are studied since they are commonly considered as surrogates of high-pressure fuel/air mixtures for gas turbines and diesel engines. ${ }^{8,9}$

The details of the computation of phase envelopes will be omitted here as they are a classical subject treated in reference textbooks. ${ }^{10}$ In general, vapor-liquid equilibrium curves are obtained by computing solutions of the zeroed second-order variation $\sum_{i=1}^{N} \sum_{j=1}^{N}\left(\partial^{2} F / \partial n_{i} \partial n_{j}\right)_{T, \rho, n_{k \neq i, j}} \Delta n_{i} \Delta n_{j}=0$ of the Helmholtz free energy $F$, where $\Delta n_{i}$ are non-zero perturbations of moles of species $i, \rho$ is the density, and $N$ is the number of components of the mixture ( $N=2$ in this example). Similarly, the calculation of the critical line, which corresponds to the curve connecting the critical points for different mixture compositions, follows the methodology introduced in Ref. ${ }^{11}$ The critical point of multi-component mixtures is the thermodynamic state at which the bubble point and the dew point converge (which does not necessarily occur at inflection points of isobars), and therefore corresponds to a stable point at the limit of thermodynamic stability. They are obtained from the solutions to the equation $\sum_{i=1}^{N} \sum_{j=1}^{N} \sum_{k=1}^{N}\left(\partial^{3} F / \partial n_{i} \partial n_{j} \partial n_{k}\right)_{T, \rho, n_{\ell \neq i, j, k}} \Delta n_{i} \Delta n_{j} \Delta n_{k}=0$ for the third-order variation of $F$.

The equations described above are supplemented with the Peng-Robinson ${ }^{12}$ equation of state, which is formally introduced later in Eq. (10). The coefficients $a$ and $b$ of the equation of state depend on the critical temperatures, critical pressures and acentric factors of the individual mixture components, as well as on the local temperature and mixture composition. They are obtained by first computing the individual values of the coefficients for each species, $a_{i}$ and $b_{i}$, as specified in Ref., ${ }^{13}$ which are combined using van der Waals mixing rules as

$$
a=\sum_{i=1}^{N} \sum_{j=1}^{N} X_{i} X_{j} a_{i j}, \quad b=\sum_{i=1}^{N} X_{i} b_{i} \quad \text { with } \quad a_{i j}=\left(1-\eta_{i j}\right) \sqrt{a_{i} a_{j}},
$$

where $X_{i}$ is the molar fraction of species $i$, and $\eta_{1,2}=0.1561$ is a binary interaction parameter fitted to experimental data in Ref. ${ }^{14}$

The vapor-liquid equilibrium curves resulting from the computations are shown in Figure 1 in a threedimensional space formed by $P, T$ and the mass fraction of n-dodecane $Y$. The three-dimensional twophase region, which is enclosed under the surface enveloping the curves, reaches much larger pressures than the critical pressure of each component. In practical terms, this is translated into the fact that an n-dodecane liquid jet flowing into a nitrogen ambient at pressures much larger than $P_{c, 1}=18$ bar may 


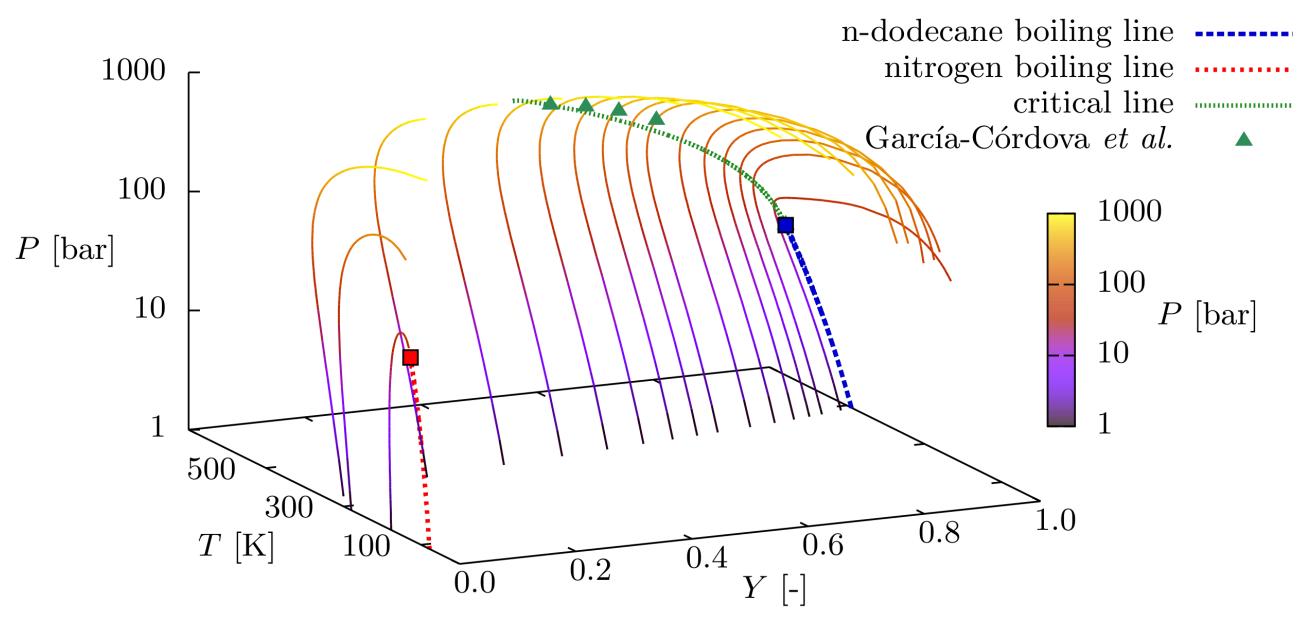

Figure 1. Vapor-liquid equilibrium curves (solid lines) for n-dodecane/nitrogen mixtures colored by pressure, with $Y$ indicating the mass fraction of n-dodecane. Pure-substance boiling lines for nitrogen (dashed red) and n-dodecane (dashed blue) are shown, along with their corresponding critical points (squares). Experimentally measured critical points are denoted by triangles.

undergo transcritical trajectories that cross the two-phase region. As a result, such flow may display remnant effects of surface tension and atomization characteristics similar to lower pressure jets that in principle were not expected to be observed in these thermodynamic conditions, as shown in experiments in Ref. ${ }^{8}$ This critical-point elevation property is also illustrated by the divergence of the the critical line arriving to the nitrogen side, as observed in Figure 2, which indicates that the two-phase region is unbounded in pressure. Conversely, the critical line starting at the nitrogen critical point meets a liquid-liquid-gas phase-equilibrium line (indistinguishable from the nitrogen boiling line in the scales of Figure 2) at an upper critical end point. The three-phase equilibrium line continues toward lower pressures and temperatures between the boiling lines of the two pure components, thereby suggesting that the crossing of the three-phase equilibrium region is only relevant at pressures lower than $P_{c, 2}=34$ bar and across a very narrow range of temperatures around $T_{c, 2}=126 \mathrm{~K}$. The phenomena of divergence of the critical line and occurrence of three-phase equilibria are typical in mixtures classified as class-II/type-III according to the analysis in Ref. ${ }^{15}$ This group of mixtures, to which other n-alkane/nitrogen systems also belong, is characterized by individual components with very different critical temperatures.

It should be stressed that the computation of critical points in complex mixtures involves a number of assumptions and model parameter values that find little justification on physical grounds. For instance, the mixing rules (1) correspond to an ad-hoc molar weighting of the individual coefficients $a_{i}$ and $b_{i}$, whose expressions depend on calibrated interaction parameters and measured critical points of the pure substances. ${ }^{13}$ However, it is of some interest to note that the resulting divergent trend of the critical line computed from the vapor-liquid equilibrium agrees well with the values experimentally obtained in Ref., ${ }^{14}$ as shown in Figures 1 and 2 .

The phase diagram facilitates the understanding of the thermodynamic trajectories involved in the injection of hydrocarbon fuels into high-pressure environments. As an illustration, consider the examples of linear thermodynamic trajectories followed by mixture elements in the problem of a liquid n-dodecane jet injected in a nitrogen environment at $900 \mathrm{~K}$, which are provided in Figure 3. Two fuel injection temperatures, corresponding to $363 \mathrm{~K}$ (case 1) and $563 \mathrm{~K}$ (case 2), are studied, along with three nitrogen-environment pressures, namely, 50,100, and 200 bar. The trajectories are superimposed on maximum-temperature curves bounding the two-phase region at each pressure. Note that the trajectories resulting from integration of the conservation equations may not be generally linear. ${ }^{16}$

For all pressure values considered in case 1, the mixture elements start as compressed liquids in the n-dodecane stream. As heat is supplied from the surrounding gas, the mixture elements enter the two-phase region where they necessarily separate into liquid and vapor phases by an interface where surface-tension forces operate. The mixture elements eventually exit the two-phase region and change phase to a supercritical state while mixing with the surrounding nitrogen gas. On the other hand, in case 2 , the intersection with the two-phase region is completely avoided for the largest pressure value considered in the nitrogen environment. 


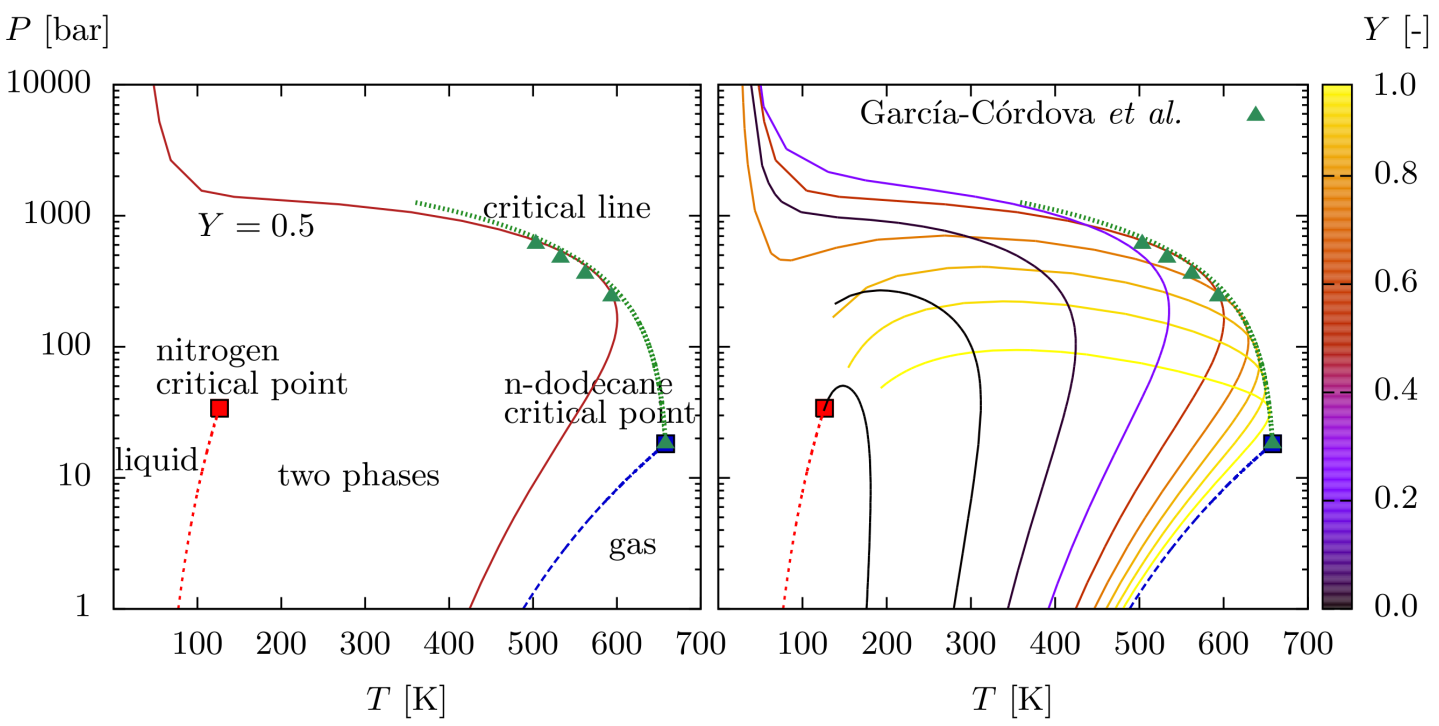

Figure 2. Two-dimensional projections of the vapor-liquid equilibrium curves on the temperature-pressure plane for a n-dodecane/nitrogen system, including description of the diagram for $Y=0.5$ (left panel) along with arrays of vaporequilibrium curves for different values of $Y$ (right panel). Refer to caption in Figure 1 for the remaining symbols and lines.

The resulting thermodynamic path involves no sustained separation of the two components through an interface. In these conditions, atomization and mixing are solely limited by the rate of supercritical mass diffusion.

Figure 3 demonstrates that the fluid dynamical description of problems where transcritical conditions are traversed requires the treatment of emerging and vanishing interfaces in the same flow field depending on the local thermodynamic conditions of the mixture elements as they move across the flow field. A set of modifications to the Navier-Stokes equations are presented below that attempt to enable this treatment.

\section{Conservation equations for transcritical flows}

This section describes a general formulation of the conservation equations and associated transport fluxes based on diffuse-interface theory for multi-component flows. The development begins by outlining the main characteristics of the theory, and continues with the general conservation equations along with derivations of the transport fluxes from thermodynamic considerations.

\section{III.A. Non-local thermodynamic effects}

The theoretical foundations of the diffuse-interface approach were first established for single-component systems in thermodynamic equilibrium by van der Waals. ${ }^{17}$ It was later extended to study binary mixtures near the critical point by Cahn and Hilliard. ${ }^{18}$ More recently, the approach has been coupled to the equations of fluid motion for single-component systems. ${ }^{19}$ Thermodynamic investigations of transition between twoand single-phase states using the diffuse-interface approach for inert and chemically reacting multi-component systems have also provided understanding of the behavior of propellants in high-pressure environments. ${ }^{7,20}$

The diffuse-interface theory rests upon the fact that the two-phase region within the liquid-vapor equilibrium curve in the phase diagram is thermodynamically unstable, in that no stable thermodynamic state exists that describes a spatially homogeneous mixture of liquid and vapor. This is perhaps easily visualized by bringing a single-component fluid in a closed vessel to its vapor pressure. The substance tends to separate in two phases of unequal density bounded by a thin transition layer where capillary forces become important. The general results of the diffuse-interface theory are aimed at describing the mechanics of the transition layer as well as the fluxes of energy and mass across it. The former correspond to familiar surface-tension forces emerging from the resistance of the interface to get deformed, while the latter represent vaporization and diffusive mixing. 


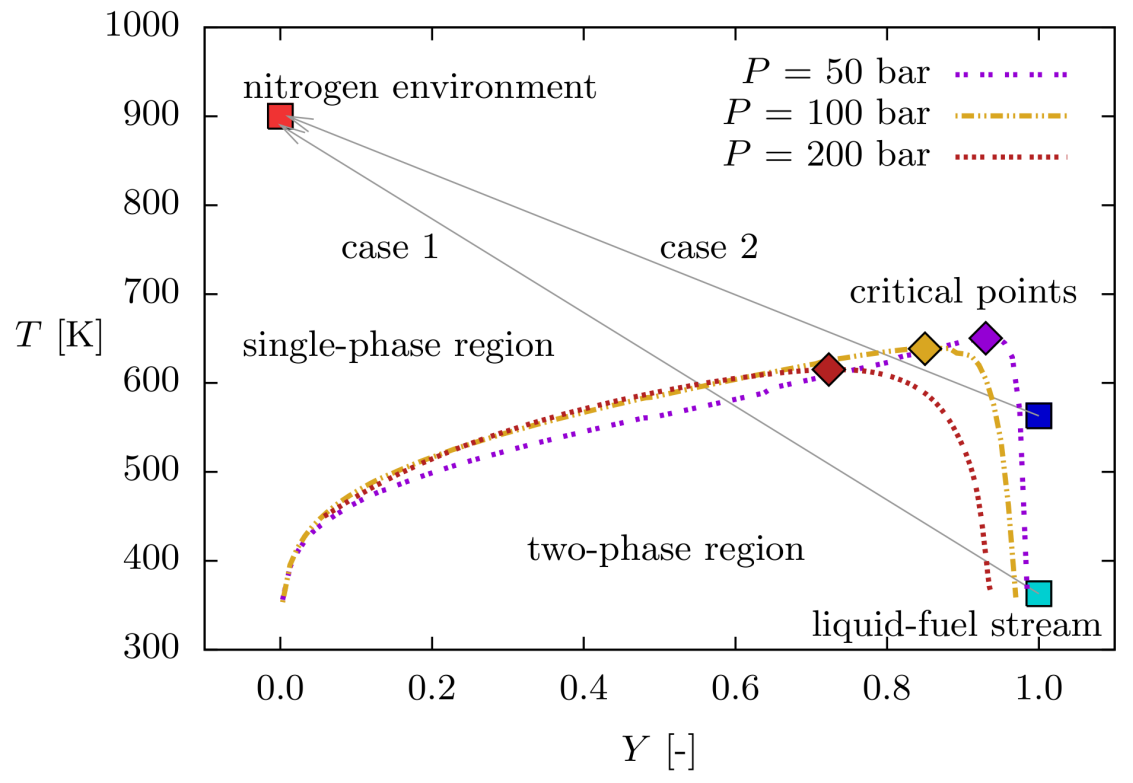

Figure 3. Examples of linear thermodynamic trajectories (solid lines) superimposed on maximum-temperature curves (dashed colored lines) bounding the two-phase region of n-dodecane/nitrogen mixtures at nitrogen-environment pressures of 50,100, and 200 bar. Symbols denote the thermodynamic conditions of the nitrogen environment (left-hand-side square) and n-dodecane stream at $363 \mathrm{~K}$ (lower right-hand-side square) and $663 \mathrm{~K}$ (upper right-hand-side square). The diamond symbols represent critical points at the corresponding pressure.

The description of the structure of the transition layer requires consideration of non-local thermodynamic potentials, where the non-locality is represented by gradients of selected variables. Additional considerations based on the second principle of thermodynamics typically preclude non-locality to be expressed only in terms of composition or density gradients (e.g., see Ref. ${ }^{21}$ for details on the mathematical justification). The analysis is facilitated when the degree of non-locality is assumed to be small, with the characteristic length of the composition gradients being large compared to intermolecular distances, which typically limits the theory to situations when the interface is relatively thick compared to the molecular mean free path, as in conditions near and above the critical point. In this limit, the disturbances of the local thermodynamic state are proportional to the square of the composition gradients in the first approximation. ${ }^{18}$ For instance, the non-local corrections to the specific values of Helmholtz free energy $f$, internal energy $e$ and entropy $s$ are

$$
\begin{gathered}
f_{\mathrm{NL}}=f+\frac{1}{2 \rho} \sum_{i=1}^{N} \sum_{j=1}^{N} \kappa_{i j} \nabla \rho_{i} \nabla \rho_{j}, \quad e_{\mathrm{NL}}=e+\frac{1}{2 \rho} \sum_{i=1}^{N} \sum_{j=1}^{N} \kappa_{i j}^{e} \nabla \rho_{i} \nabla \rho_{j}, \\
s_{\mathrm{NL}}=s+\frac{1}{2 \rho} \sum_{i=1}^{N} \sum_{j=1}^{N} \kappa_{i j}^{s} \nabla \rho_{i} \nabla \rho_{j},
\end{gathered}
$$

where $\rho$ is the mixture density, $\rho_{i}$ is the partial density of species $i$, and $N$ is the number of species. Additionally, $\kappa_{i j}, \kappa_{i j}^{e}$ and $\kappa_{i j}^{s}$ are gradient-energy coefficients, which can be computed directly as a function of collision parameters from kinetic-theory considerations of interactions between molecules in regions subjected to macroscopic density gradients (e.g., see Ref. ${ }^{22}$ and Chapter 1 in Ref. ${ }^{23}$ ).

Since the gradient-energy coefficients $\kappa_{i j}$ are related to the interface thickness and surface tension, their precise characterization is central to the predictions of the diffuse-interface theory. However, appropriate formulations of this parameters are lacking, and most investigations utilize relations of the type $\kappa_{i j}=$ $\sqrt{\kappa_{i i} \kappa_{j j}}=\kappa_{j i}$ for the cross-influence coefficients $i \neq j$, along with empirical correlations for the individual coefficients $\kappa_{i i}$ such as ${ }^{24}$

$$
\ln \left(\frac{\kappa_{i i}}{a_{i} b_{i}{ }^{2 / 3}} N_{A}^{8 / 3}\right)=\kappa_{0, i}+\kappa_{1, i} \ln \left(1-\frac{T}{T_{c, i}}\right)+\kappa_{2, i}\left[\ln \left(1-\frac{T}{T_{c, i}}\right)\right]^{2}
$$


for $T / T_{c, i} \leq 0.95$, where $T_{c, i}$ is the critical temperature value, and $N_{A}$ is the Avogadro's number. In Eq. (3), the correlation coefficients $\kappa_{0, i}, \kappa_{1, i}$ and $\kappa_{2, i}$ are usually calibrated based on experimental measurements of surface tension, while the parameters $a_{i}$ and $b_{i}$ correspond to coefficients of the equation of state, as described in Section II. Note that models such as Eq. (3) typically yield $\kappa_{i i}=0$ above the critical temperature of the corresponding species, as suggested by the fact that the surface tension vanishes for single-component systems above the critical point. For instance, in the n-dodecane/nitrogen system described in Section II, the relevant gradient-energy coefficient becomes that of the $\mathrm{n}$-dodecane, $\kappa_{1,1}$, since the temperature in the flow is larger than the critical temperature of nitrogen everywhere (i.e., $\kappa_{2,2}=\kappa_{1,2}=\kappa_{2,1}=0$ ).

Exact expressions relating the gradient-energy coefficients $\kappa_{i j}, \kappa_{i j}^{e}$ and $\kappa_{i j}^{s}$ can be easily derived by substituting the relations (2) into the definition of the local Helmholtz free energy, $f=e-T s$, with $s=-(\partial f / \partial T)_{\rho, n_{i}}$, thereby yielding

$$
\kappa_{i j}^{e}=\kappa_{i j}+T\left(\frac{\partial \kappa_{i j}}{\partial T}\right)_{\rho, n_{k}} \text { and } \kappa_{i j}^{s}=-\left(\frac{\partial \kappa_{i j}}{\partial T}\right)_{\rho, n_{k}} .
$$

The consideration of non-local thermodynamic potentials, as in Eqs. (2)-(4), leads to the emergence of interface-related transport fluxes and mechanical stresses in the conservation equations as described below.

\section{III.B. Conservation equations}

The description of thin interfaces and their dynamics in conjunction with the outer fluid motion in a single Eulerian field requires non-trivial extensions of the Navier-Stokes conservation equations. In principle, the derivation of these modifications from molecular considerations and first principles is a difficult task due to the lack of a clear physical understanding of the molecular structure of fluids across the critical point. In this study, a phenomenological approach is followed based on a linear augmentation of the deviatoric part of the stress tensor, $\boldsymbol{\tau}$, and the heat and species diffusion fluxes, $\boldsymbol{q}$ and $\boldsymbol{J}_{\boldsymbol{i}}$, with the corresponding interfacial transport terms $\mathcal{K}, \mathcal{Q}$ and $\mathcal{J}_{\boldsymbol{i}}$ derived from the diffuse-interface theory. These, as shown below, can be made to satisfy certain conditions of entropy maximization that are in accord with the second law of thermodynamics. The resulting conservation equations for mass, momentum, species and total energy are

$$
\begin{gathered}
\frac{\partial \rho}{\partial t}+\nabla \cdot(\rho \mathbf{v})=0 \\
\frac{\partial(\rho \mathbf{v})}{\partial t}+\nabla \cdot(\rho \mathbf{v} \otimes \mathbf{v})=-\nabla P_{\mathrm{NL}}+\nabla \cdot(\boldsymbol{\tau}+\mathcal{K}), \\
\frac{\partial\left(\rho Y_{i}\right)}{\partial t}+\nabla \cdot\left(\rho \mathbf{v} Y_{i}\right)=-\nabla \cdot\left(\boldsymbol{J}_{\boldsymbol{i}}+\mathcal{J}_{\boldsymbol{i}}\right), \quad i=1, \ldots, N, \\
\frac{\partial(\rho E)}{\partial t}+\nabla \cdot(\rho \mathbf{v} E)=-\nabla \cdot\left(P_{\mathrm{NL}} \mathbf{v}\right)-\nabla \cdot(\boldsymbol{q}+\mathcal{Q})+\nabla \cdot[(\boldsymbol{\tau}+\mathcal{K}) \cdot \mathbf{v}],
\end{gathered}
$$

which describe the continuum dynamics of a multi-phase, multi-component fluid of $N$ species that moves at a mass-averaged velocity $\mathbf{v}$ and has a local density $\rho$ and total energy $E$, and which may contain thin interfaces separating different phases. In this formulation, $Y_{i}$ is the mass fraction of species $i, \boldsymbol{q}=\boldsymbol{q}_{\boldsymbol{c}}+\sum_{i=1}^{N} h_{i} \boldsymbol{J}_{\boldsymbol{i}}$ is the sum of the heat conduction and the energy flux by inter-diffusion, $h_{i}$ is the partial specific enthalpy, and $P_{\mathrm{NL}}$ a non-local thermodynamic pressure defined as

$$
P_{\mathrm{NL}}=P-\frac{1}{2} \sum_{i=1}^{N} \sum_{j=1}^{N} \kappa_{i j} \nabla \rho_{i} \nabla \rho_{j} .
$$

The convenience of redefining pressure as in Eq. (9), will become clear in Section III.C. In Eq. (9) the local thermodynamic pressure $P$ can be obtained, for instance, from the cubic equation of state ${ }^{12}$

$$
P=\frac{\mathrm{R}^{0} T}{v-b}-\frac{a}{v^{2}+2 b v-b^{2}},
$$

whose utilization is beneficial at the high pressures considered here. In the notation, $v=W / \rho$ is the molar volume, with $W=\left(\sum_{i=1} Y_{i} / W_{i}\right)^{-1}$ the mean molecular weight. The coefficients $a$ and $b$, which correspond to mixture-averaged versions of the pure-substance ones $a_{i}$ and $b_{i}$ as in Eq. (1), account for real-gas effects 
such as finite packing and increased intermolecular interactions at large densities and pressures. It should be stressed that the choice of Eq. (10) is not central to the diffuse-interface formalism insofar as it reproduces the multivalued character of the mixture density in conditions of phase change. Note that several other equations of state are available in the literature that have similar characteristics. ${ }^{25,26}$

Chemical conversion sources have been excluded for simplicity from Eq. (7). Gas-phase combustion reactions tend to occur far from interfaces and in regions where the local mass fraction of fuel vapor is sufficiently small to warrant stoichiometric proportions. However, this approximation may not be appropriate if thermal decomposition of the liquid fuel plays an important role in modifying the interface properties.

In the species conservation equation (7), the different $N$ components of the mixture are described by their corresponding mass fractions irrespectively of their phase state. Note that this is in contrast with traditional treatments of dispersed multi-phase flows, where the gas and liquid mass fractions are described by their corresponding conservation equations. In the diffuse-interface formulation, the phases are separated by interfaces in thermodynamic conditions corresponding to the two-phase region. In those situations, the interfacial stress tensor $\mathcal{K}$ in the momentum equation (6) provides information about the dynamical equilibrium of the separating interface, while the fluxes $\mathcal{Q}$ and $\mathcal{J}_{\boldsymbol{i}}$ modify the transport of heat and mass across the interface accordingly. The high-pressure characteristics of the transport fluxes are described in detail in Section III.C.

A complete description of the mixture state requires specification of the analytical form of the thermodynamic potentials. At high pressures, increasing departures from the ideal-gas theory are observed, and consequently, derivation of more complex expressions are necessary. A common approach to express highpressure real-gas thermodynamic potentials is to decompose them into the sum of their ideal-gas counterparts (denoted below by the superscript ${ }^{0}$ ) and departure functions that measure deviations with respect to the ideal-gas behavior. ${ }^{28}$ For instance, the departure function for the molar enthalpy is

$$
\bar{h}-\bar{h}^{0}=\int_{T^{0}}^{T} C_{p}^{0} d T+\int_{0}^{P}\left[v-T\left(\frac{\partial v}{\partial T}\right)_{P}\right] d P,
$$

where $\bar{h}^{0}$ and $C_{p}^{0}$ are the ideal-gas reference molar values of enthalpy and constant-pressure heat capacity, with $T^{0}=298.15 \mathrm{~K}$. Subsequently, the molar internal energy can be obtained from the enthalpy definition as

$$
\bar{e}=\bar{h}-P v .
$$

These expressions are valid for any equation of state. Exact forms of the departure functions for multi-species mixtures can be found in Ref. ${ }^{29}$ for the Peng-Robinson equation of state.

Similar considerations apply for the molar chemical potential

$$
\bar{\mu}_{i}=\left(\frac{\partial G}{\partial n_{i}}\right)_{T, P, n_{j \neq i}}
$$

defined as the partial molar of the Gibbs free energy $G$. The corresponding decomposition is given by

$$
\bar{\mu}_{i}=\bar{\mu}_{i}^{0}+\mathrm{R}^{0} T \ln \varphi_{i},
$$

where $\bar{\mu}_{i}^{0}(T, P)$ is the ideal-gas counterpart. In Eq. (14), the departure function involves the dimensionless fugacity coefficient $\varphi_{i}=f_{i} /\left(X_{i} P\right)$, which represents the ratio of the fugacity $f_{i}$ to the partial pressure. In particular, for the Peng-Robinson equation of state, the logarithm of the fugacity coefficient becomes

$$
\ln \varphi_{i}=\frac{b_{i}}{b}(Z-1)-\ln (Z-B)-\frac{A}{2 \sqrt{2} B}\left[\frac{2 \sum_{j=1}^{N} X_{i} a_{i j}}{a}-\frac{b_{i}}{b}\right] \ln \left[\frac{Z+(1+\sqrt{2}) B}{Z+(1-\sqrt{2}) B}\right],
$$

where $A=a P /\left(\mathrm{R}^{0} T\right)^{2}, B=b P /\left(\mathrm{R}^{0} T\right)$, and the coefficients $a$ and $b$ are given by Eq. (1). Additionally, $Z=P v /\left(\mathrm{R}^{0} T\right)$ is the compressibility factor, which quantifies the departures from the reference value $Z=1$ corresponding to the ideal-gas equation of state. Figure 4 shows the fugacity coefficients for an ndodecane/nitrogen mixture at high pressure. While departures from ideality are largest at low temperatures, the chemical potential resembles that of the ideal gas for sufficiently large temperatures (e.g., above $900 \mathrm{~K}$ ). Similar trends hold up to pressures of order $10^{3}$ bar.

The transport coefficients also undergo large variations across the phase diagram at high pressures. The transition from liquid-like to gas-like characteristics prevent the utilization of simple expressions for the 

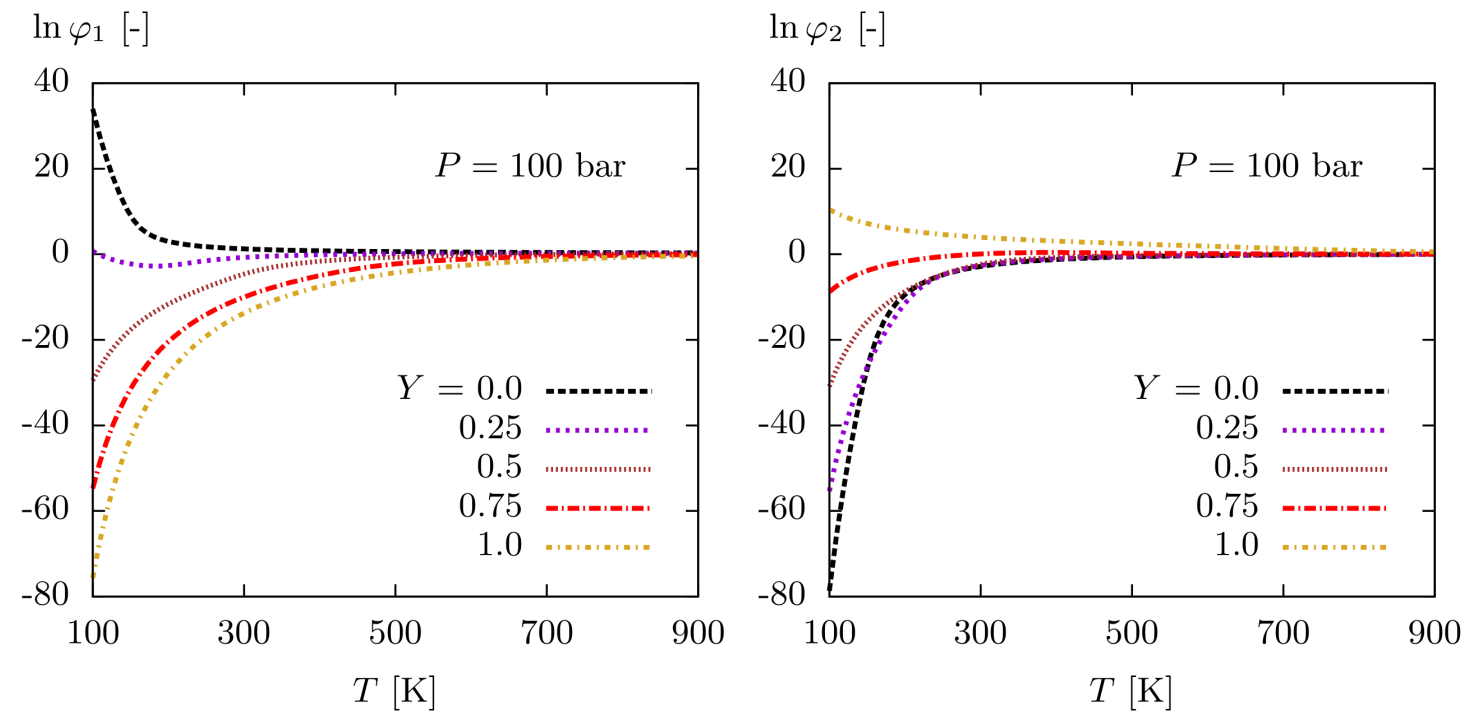

Figure 4. Logarithm of the n-dodecane (left panel) and nitrogen (right panel) fugacity coefficients as a function of temperature and fuel mass fraction for an n-dodecane/nitrogen mixture at $P=100$ bar.

evaluation of mixture's viscosity, thermal conductivity and diffusion coefficients. Instead, the method in Ref. $^{27}$ is typically used to evaluate viscosity and thermal conductivity as function of $T$ and $\rho$, whereas diffusion coefficients can be calculated, for example, following the expressions given in Chapter 11 in Ref. ${ }^{28}$ for high-pressure conditions. These coefficients, however, are currently subject to large uncertainties.

\section{III.C. Transport fluxes}

The system of conservation equations (5)-(8) requires closure expressions for $\boldsymbol{\tau}, \mathcal{K}, \boldsymbol{q}_{\boldsymbol{c}}, \mathcal{Q}, \boldsymbol{J}_{\boldsymbol{i}}$, and $\mathcal{J}_{\boldsymbol{i}}$. This is achieved through the method of irreversible thermodynamics by specifying constitutive relations such that the entropy production is non-negative. This methodology requires that one finds the conservation equation of entropy guided by the fact that the source terms are written as a sum of products of fluxes and thermodynamic forces. ${ }^{31}$ The formulation is greatly simplified when $\kappa_{i j}$ does not depend on temperature, in such a way that $\kappa_{i j}^{e}=\kappa_{i j}$ and $\kappa_{i j}^{s}=0$, as implied by Eq. (4). In view of the experimental correlation (3), this is an approximation that has an unclear physical justification but has however been used in the literature $^{22,30}$ and will also be followed here. Starting from the second principle of thermodynamics for a multi-component system

$$
T d s=d e+P d(1 / \rho)-\sum_{i=1}^{N}\left(\bar{\mu}_{i} / W_{i}\right) d Y_{i}
$$

and substituting the relations (2), the equation

$$
T d s_{\mathrm{NL}}=d e_{\mathrm{NL}}+P_{\mathrm{NL}} d(1 / \rho)-\sum_{i=1}^{N}\left(\bar{\mu}_{i} / W_{i}\right) d Y_{i}-\sum_{i=1}^{N} \boldsymbol{\psi}_{\boldsymbol{i}} \cdot d\left(\nabla \rho_{i}\right) / \rho
$$

is obtained, where

$$
\boldsymbol{\psi}_{\boldsymbol{i}}=\sum_{j=1}^{N} \kappa_{i j} \nabla \rho_{j}
$$

is an auxiliary variable. A transport equation for the specific entropy $s_{\mathrm{NL}}$ can be derived by taking the material derivative of Eq. (17) and substituting the conservation equations (5)-(8) into the resulting expression, which yields

$$
\rho \frac{D s_{\mathrm{NL}}}{D t}+\nabla \cdot\left\{\frac{1}{T}\left[\boldsymbol{q}_{\boldsymbol{c}}+\mathcal{Q}-\sum_{i=1}^{N}\left\{\boldsymbol{\psi}_{\boldsymbol{i}}\left[\rho_{i} \nabla \cdot \boldsymbol{v}+\nabla \cdot\left(\boldsymbol{J}_{\boldsymbol{i}}+\mathcal{J}_{\boldsymbol{i}}\right)\right]-T \widetilde{s}_{i} \boldsymbol{J}_{\boldsymbol{i}}+\widetilde{\mu}_{i} \mathcal{J}_{\boldsymbol{i}}\right\}\right]\right\}=\dot{s}_{\text {prod }}
$$


where $\widetilde{s}_{i}=s_{i}+\left(\nabla \cdot \boldsymbol{\psi}_{\boldsymbol{i}}\right) / T$ and $\widetilde{\mu}_{i}=\mu_{i}-\nabla \cdot \boldsymbol{\psi}_{\boldsymbol{i}}$ are generalized versions of the partial specific entropy and specific chemical potential, respectively, and $\dot{s}_{\text {prod }}$ is an entropy source given by

$$
\begin{aligned}
\dot{s}_{\text {prod }}= & \frac{1}{T}\left[\boldsymbol{\tau}+\mathcal{K}-\sum_{i=1}^{N}\left(\rho_{i} \nabla \cdot \boldsymbol{\psi}_{\boldsymbol{i}} \boldsymbol{I}-\nabla \rho_{i} \otimes \boldsymbol{\psi}_{\boldsymbol{i}}\right)\right]: \nabla \boldsymbol{v} \\
& +\left[\boldsymbol{q}_{\boldsymbol{c}}+\mathcal{Q}-\sum_{i=1}^{N}\left\{\boldsymbol{\psi}_{\boldsymbol{i}}\left[\rho_{i} \nabla \cdot \boldsymbol{v}+\nabla \cdot\left(\boldsymbol{J}_{\boldsymbol{i}}+\mathcal{J}_{\boldsymbol{i}}\right)\right]-T \widetilde{s}_{i}\left(\boldsymbol{J}_{\boldsymbol{i}}+\mathcal{J}_{\boldsymbol{i}}\right)+h_{i} \mathcal{J}_{\boldsymbol{i}}\right\}\right] \cdot \nabla\left(\frac{1}{T}\right) \\
& -\frac{1}{T} \sum_{i=1}^{N}\left(\boldsymbol{J}_{\boldsymbol{i}}+\mathcal{J}_{\boldsymbol{i}}\right) \cdot \nabla \widetilde{\mu}_{i},
\end{aligned}
$$

with $\boldsymbol{I}$ the identity matrix. In the derivation of Eq. (20), use of the thermodynamic relation $T s_{i}=h_{i}-\mu_{i}$ and of the vector identity

$$
\boldsymbol{\psi}_{\boldsymbol{i}} \cdot \frac{D}{D t}\left(\nabla \rho_{i}\right)=\boldsymbol{\psi}_{\boldsymbol{i}} \cdot \nabla\left(\frac{D \rho_{i}}{D t}\right)-\left(\nabla \rho_{i} \otimes \boldsymbol{\psi}_{\boldsymbol{i}}\right): \nabla \mathbf{v}
$$

have been made. Similarly, the gradient of the specific chemical potential in the last term of Eq. (20) can be expanded as $\nabla \widetilde{\mu}_{i}=\nabla_{T} \widetilde{\mu}_{i}-\left\{s_{i}+\left[\partial\left(\nabla \cdot \boldsymbol{\psi}_{\boldsymbol{i}}\right) / \partial T\right]_{P, n_{j}}\right\} \nabla T$, where the reciprocity relation $\left(\partial \mu_{i} / \partial T\right)_{P, n_{j}}=-s_{i}$ has been used, with the subindex $T_{T}$ indicating variations at constant temperature. Upon substituting this relation along with the species diffusion-flux conservation $\sum_{i=1}^{N}\left(\boldsymbol{J}_{\boldsymbol{i}}+\mathcal{J}_{\boldsymbol{i}}\right)=0$ into Eq. (20), the entropy source becomes

$$
\begin{aligned}
\dot{s}_{\text {prod }}= & \frac{1}{T}\left[\boldsymbol{\tau}+\mathcal{K}-\sum_{i=1}^{N}\left(\rho_{i} \nabla \cdot \boldsymbol{\psi}_{\boldsymbol{i}} \boldsymbol{I}-\nabla \rho_{i} \otimes \boldsymbol{\psi}_{\boldsymbol{i}}\right)\right]: \nabla \boldsymbol{v} \\
& +\left[\boldsymbol{q}_{\boldsymbol{c}}+\mathcal{Q}-\sum_{i=1}^{N}\left\{\boldsymbol{\psi}_{\boldsymbol{i}}\left[\rho_{i} \nabla \cdot \boldsymbol{v}+\nabla \cdot\left(\boldsymbol{J}_{\boldsymbol{i}}+\mathcal{J}_{\boldsymbol{i}}\right)\right]-\chi_{i}\left(\boldsymbol{J}_{\boldsymbol{i}}+\mathcal{J}_{\boldsymbol{i}}\right)+h_{i} \mathcal{J}_{\boldsymbol{i}}\right\}\right] \cdot \nabla\left(\frac{1}{T}\right) \\
& +\sum_{i=1}^{N-1}\left(\boldsymbol{J}_{\boldsymbol{i}}+\mathcal{J}_{\boldsymbol{i}}\right) \cdot\left[-\nabla_{T}\left(\widetilde{\mu}_{i}-\widetilde{\mu}_{N}\right)\left(\frac{1}{T}\right)\right]
\end{aligned}
$$

where $\chi_{i}=\nabla \cdot \boldsymbol{\psi}_{\boldsymbol{i}}-T\left[\partial\left(\nabla \cdot \boldsymbol{\psi}_{\boldsymbol{i}}\right) / \partial T\right]_{P, n_{j}}$ is an auxiliary variable.

The entropy source (22) consists of three terms represented by each one of the lines. They represent entropy production by three different sources: flow gradients, temperature gradients, and gradients of the chemical potential at constant temperature. Following the methodology of Onsager ${ }^{32}$ (see also Landau \& Lifshitz $^{31}$ ), the terms $\mathcal{K}, \mathcal{Q}$ and $\mathcal{J}_{\boldsymbol{i}}$ can be computed as follows. Each term of the entropy source can be represented as a product of a flux $\phi_{\boldsymbol{i}}$ and a thermodynamic force $\boldsymbol{F}_{\boldsymbol{i}}$ as $\dot{s}_{\text {prod }}=\sum_{i=1}^{N} \boldsymbol{\phi}_{\boldsymbol{i}} \cdot \boldsymbol{F}_{\boldsymbol{i}}$. In the case of dissipative systems, the second law of thermodynamics requires the entropy production to be non-negative, $\dot{s}_{\text {prod }} \geq 0$. As a consequence, the final forms of the unknown fluxes $\phi_{\boldsymbol{i}}$ must be such that they satisfy this thermodynamic requirement. One possible approach to warrant this is to assume linear relations between fluxes and forces as $\phi_{\boldsymbol{i}}=\sum_{k=1}^{N} \mathcal{L}_{i k} \boldsymbol{F}_{\boldsymbol{k}}$, where $\mathcal{L}_{i k}$ are phenomenological coefficients that are reciprocal, $\mathcal{L}_{i k}=\mathcal{L}_{k i}$, and positive diagonal, $\mathcal{L}_{i i}>0$.

The number of couplings between the different types of fluxes is reduced by means of the Curie principle ${ }^{33}$ which states that the fluxes and thermodynamic forces of different tensorial character do not couple. This principle implies that the viscous stresses depend exclusively on $\nabla \boldsymbol{v}$, while the expressions of the diffusive fluxes of heat and species are only function of the gradients of temperature and chemical potential. For instance, in the case of Newtonian fluids, the expression for the viscous stresses corresponds to $\boldsymbol{\tau}=\eta\left(\nabla \boldsymbol{v}+\nabla \boldsymbol{v}^{T}\right)+(\zeta-2 \eta / 3)(\nabla \cdot \boldsymbol{v}) \boldsymbol{I}$ where $\eta$ and $\zeta$ are the shear and bulk viscosities, respectively. Accordingly, the viscous dissipation $\boldsymbol{\tau}: \nabla \boldsymbol{v}$ is always a positive source of entropy in Eq. (22).

The interfacial stress $\mathcal{K}$ is assumed to be an elastically restoring one, in such a way that the first term in Eq. (22) yields zero-production of entropy for interface-related terms. As a result, the interfacial stress tensor becomes

$$
\mathcal{K}=\sum_{i=1}^{N}\left(\rho_{i} \nabla \cdot \boldsymbol{\psi}_{\boldsymbol{i}} \boldsymbol{I}-\nabla \rho_{i} \otimes \boldsymbol{\psi}_{\boldsymbol{i}}\right)
$$


Although the surface tension does not appear explicitly in Eq. (23), its effects are accounted for in the density gradients. For a quasi-planar interface, an effective surface-tension coefficient can be defined as

$$
\sigma=\sum_{i=1}^{N} \sum_{j=1}^{N} \int_{-\infty}^{+\infty} \kappa_{i j} \frac{d \rho_{i}}{d \xi} \frac{d \rho_{j}}{d \xi} d \xi
$$

which, in view of Eq. (2), is proportional to the excess of free energy contained in the interface, where $\xi$ is the coordinate normal to the interface. ${ }^{24}$

The interfacial heat flux is cast into the form

$$
\mathcal{Q}=\sum_{i=1}^{N}\left\{\boldsymbol{\psi}_{\boldsymbol{i}}\left[\rho_{i} \nabla \cdot \boldsymbol{v}+\nabla \cdot\left(\boldsymbol{J}_{\boldsymbol{i}}+\mathcal{J}_{\boldsymbol{i}}\right)\right]-\chi_{i}\left(\boldsymbol{J}_{\boldsymbol{i}}+\mathcal{J}_{\boldsymbol{i}}\right)+h_{i} \mathcal{J}_{i}\right\}+\boldsymbol{\Theta} .
$$

The term $\Theta$ is a dissipating component that produces entropy and is computed below.

In order to obtain expressions for the diffusive fluxes of heat and species, it is convenient to express Eq. (22) in the matrix flux-force form

$$
\dot{s}_{\text {prod }}=\left[L_{q q} \boldsymbol{F}_{1}+\sum_{k=1}^{N-1} L_{q k} \boldsymbol{F}_{k+1}\right] \cdot \boldsymbol{F}_{1}+\sum_{i=1}^{N-1}\left[L_{i q} \boldsymbol{F}_{1}+\sum_{k=1}^{N-1} L_{i k} \boldsymbol{F}_{k+1}\right] \cdot \boldsymbol{F}_{i+1},
$$

where $L_{q q}=\mathcal{L}_{1,1}, L_{q, k-1}=\mathcal{L}_{1 k}(k=2, \ldots, N), L_{i-1, q}=\mathcal{L}_{i 1}(i=2, \ldots, N), L_{i-1, k-1}=\mathcal{L}_{i k}(i, k=2, \ldots, N)$, $\boldsymbol{F}_{1}=\nabla(1 / T)$ and $\boldsymbol{F}_{i>1}=-\nabla_{T}\left(\widetilde{\mu}_{i}-\widetilde{\mu}_{N}\right)(1 / T)$. Comparing the last two terms of Eq. (22) with Eq. (26) provides the expressions

$$
\boldsymbol{q}_{\boldsymbol{c}}=-\frac{L_{q q}}{T^{2}} \nabla T-\sum_{k=1}^{N-1} \frac{L_{q k}}{T} \nabla_{T}\left(\mu_{k}-\mu_{N}\right), \quad \boldsymbol{\Theta}=\sum_{k=1}^{N-1} \frac{L_{q k}}{T} \nabla_{T} \nabla \cdot\left(\boldsymbol{\psi}_{k}-\boldsymbol{\psi}_{N}\right),
$$

and

$$
\boldsymbol{J}_{\boldsymbol{i}}=-\frac{L_{i q}}{T^{2}} \nabla T-\sum_{k=1}^{N-1} \frac{L_{i k}}{T} \nabla_{T}\left(\mu_{k}-\mu_{N}\right), \quad \mathcal{J}_{i}=\sum_{k=1}^{N-1} \frac{L_{i k}}{T} \nabla_{T} \nabla \cdot\left(\boldsymbol{\psi}_{k}-\boldsymbol{\psi}_{N}\right),
$$

for the heat and species diffusion fluxes, respectively. Additionally, the relation $L_{q q}=\lambda T^{2}$ is obtained by analogy with Fourier's law of heat conduction, with $\lambda$ the thermal conductivity.

In Eqs. (23), (25) and (28), the tensor $\mathcal{K}$ and the fluxes $\mathcal{Q}$ and $\mathcal{J}_{\boldsymbol{i}}$, with $\boldsymbol{\Theta}$ given in Eq. (27), represent the interfacial disturbances to the deviatoric part of the stress tensor, $\boldsymbol{\tau}$, and to the diffusive fluxes of heat, $\boldsymbol{q}$ and species $\boldsymbol{J}_{\boldsymbol{i}}$, respectively. In absence of interfaces, $\mathcal{K}=\mathcal{Q}=\mathcal{J}_{\boldsymbol{i}}=0$ and $P_{\mathrm{NL}}=P$, thereby leading to a simplification of the conservation equations (5)-(8) to their classic Navier-Stokes form. Symmetries in the diffusive fluxes are illustrated by the presence of the Dufour term in $\boldsymbol{q}_{\boldsymbol{c}}$ due to chemical-potential gradients as well as the corresponding Soret term in $\boldsymbol{J}_{\boldsymbol{i}}$ due to temperature gradients.

The species diffusion flux $\boldsymbol{J}_{\boldsymbol{i}}$ can be expanded in terms of pressure and composition gradients by making use of the differential form

$$
\nabla_{T} \bar{\mu}_{k}=\left(\frac{\partial \bar{\mu}_{k}}{\partial P}\right)_{T, X_{i}} \nabla P+\sum_{j=1}^{N-1}\left(\frac{\partial \bar{\mu}_{k}}{\partial X_{j}}\right)_{T, P, X_{i \neq j}} \nabla X_{j},
$$

and the Gibbs-Duhem equation

$$
\nabla_{T} \bar{\mu}_{N}=\frac{1}{X_{N}}\left(\frac{1}{c} \nabla P-\sum_{j=1}^{N-1} X_{j} \nabla_{T} \bar{\mu}_{j}\right),
$$

with $c=\rho / W$ as the molar density. The combination of Eqs. (29)-(30) leads to the relation

$$
\begin{aligned}
\nabla_{T}\left(\mu_{k}-\mu_{N}\right)= & \sum_{\ell=1}^{N-1}\left(\frac{X_{\ell}}{W_{N} X_{N}}+\frac{\delta_{\ell k}}{W_{k}}\right) \sum_{j=1}^{N-1}\left(\frac{\partial \bar{\mu}_{\ell}}{\partial X_{j}}\right)_{T, P, X_{i \neq j}} \nabla X_{j} \\
& +\left[\frac{1}{W_{k}}\left(\frac{\partial \bar{\mu}_{k}}{\partial P}\right)_{T, X_{i}}-\frac{1}{c W_{N} X_{N}}+\sum_{\ell=1}^{N-1} \frac{X_{\ell}}{W_{N} X_{N}}\left(\frac{\partial \bar{\mu}_{\ell}}{\partial P}\right)_{T, X_{i}}\right] \nabla P
\end{aligned}
$$


for the chemical-potential gradients, where $\delta_{j k}$ is the Kronecker delta. Upon substituting Eq. (31) into Eq. (28), the species diffusion flux $\boldsymbol{J}_{\boldsymbol{i}}$ can be expressed as

$$
\boldsymbol{J}_{\boldsymbol{i}}=-\rho\left(\sum_{j=1}^{N-1} D_{i j}^{M} \nabla X_{j}+D_{i}^{T} \nabla T+D_{i}^{P} \nabla P\right),
$$

where $D_{i j}^{M}, D_{i}^{T}$ and $D_{i}^{P}$ are mass, thermal and pressure diffusion coefficients defined as

$$
\begin{aligned}
& D_{i j}^{M}=a_{i N} D_{i N} \frac{W_{i} X_{i}}{L_{i i} W} \sum_{k=1}^{N-1} L_{i k} \sum_{\ell=1}^{N-1} \frac{W_{\ell} X_{\ell}+W_{N} X_{N} \delta_{\ell k}}{W_{\ell}}\left(\frac{\partial \ln f_{\ell}}{\partial X_{j}}\right)_{T, P, X_{m \neq j}}, \\
& D_{i}^{T}=a_{i N} D_{i N} \frac{k_{i T}}{T}, \\
& D_{i}^{P}=a_{i N} D_{i N} \frac{W_{i} X_{i}}{\mathrm{R}^{0} T L_{i i} W} \sum_{k=1}^{N-1} L_{i k}\left[\frac{W_{N} X_{N}}{W_{k}} \bar{V}_{k}-\frac{1}{c}+\sum_{\ell=1}^{N-1} X_{\ell} \bar{V}_{\ell}\right],
\end{aligned}
$$

where $\left(\partial \bar{\mu}_{k} / \partial P\right)_{T, X_{i}}=\bar{V}_{k}$ and $\left(\partial \bar{\mu}_{\ell} / \partial X_{j}\right)_{T, P, X_{i \neq j}}=\mathrm{R}^{0} T \partial \ln f_{\ell} /\left.\partial X_{j}\right|_{T, P, X_{i \neq j}}$ have been used. In Eqs. (33)-

\begin{tabular}{|c|c|}
\hline Transport coefficients & \\
\hline Fickian & $D_{1,1}^{M}=a_{1,2} D_{1,2}\left(\frac{\partial \ln f_{1}}{\partial \ln X_{1}}\right)_{T, P}$ \\
\hline Barodiffusion & $D_{1}^{P}=a_{1,2} D_{1,2} \frac{X_{1}}{\mathrm{R}^{0} T}\left(\bar{V}_{1}-\frac{W_{1}}{\rho}\right)$ \\
\hline Soret & $D_{1}^{T}=a_{1,2} D_{1,2} \frac{k_{1 T}}{T}$ \\
\hline Dufour & $D_{1}^{F}=\frac{D_{1}^{T} W \mathrm{R}^{0} T}{W_{1} W_{2} X_{1}\left(1-X_{1}\right)}\left(\frac{\partial \ln f_{1}}{\partial \ln X_{1}}\right)_{T, P}$ \\
\hline Interfacial (species) & $D_{1}^{K, M}=a_{1,2} D_{1,2} \kappa_{1,1} \frac{W_{1} W_{2} X_{1}\left(1-X_{1}\right)}{W \mathrm{R}^{0} T}$ \\
\hline Interfacial (heat) & $D_{1}^{K, T}=a_{1,2} D_{1,2} \kappa_{1,1} \frac{k_{1 T}}{T}$ \\
\hline Total stress tensor & $-P_{\mathrm{NL}} \boldsymbol{I}+\boldsymbol{\tau}+\mathcal{K}$ \\
\hline Non-local pressure tensor & $-P_{\mathrm{NL}} \boldsymbol{I}=-\left(P-\frac{1}{2} \kappa_{1,1}\left|\nabla \rho_{1}\right|^{2}\right) \boldsymbol{I}$ \\
\hline Viscous stress tensor & $\boldsymbol{\tau}=\eta\left(\nabla \boldsymbol{v}+\nabla \boldsymbol{v}^{T}\right)+\left(\zeta-\frac{2}{3} \eta\right)(\nabla \cdot \boldsymbol{v}) \boldsymbol{I}$ \\
\hline Interfacial stress tensor & $\mathcal{K}=\kappa_{1,1}\left(\rho_{1} \nabla^{2} \rho_{1} \boldsymbol{I}-\nabla \rho_{1} \otimes \nabla \rho_{1}\right)$ \\
\hline Total heat flux & $q+\mathcal{Q}$ \\
\hline $\begin{array}{l}\text { Fourier, inter-diffusion } \\
\text { and Dufour heat fluxes }\end{array}$ & $\boldsymbol{q}=-\lambda \nabla T+\left(h_{1}-h_{2}\right) \boldsymbol{J}_{\mathbf{1}}-\rho D_{1}^{F} \nabla X_{1}$ \\
\hline Interfacial heat flux & $\mathcal{Q}=\kappa_{1,1} \nabla \rho_{1}\left[\rho_{1} \nabla \cdot \boldsymbol{v}+\nabla \cdot\left(\boldsymbol{J}_{\mathbf{1}}+\mathcal{J}_{\mathbf{1}}\right)\right]$ \\
\hline & $-\chi_{1}\left(\boldsymbol{J}_{1}+\mathcal{J}_{\mathbf{1}}\right)+\left(h_{1}-h_{2}\right) \mathcal{J}_{\mathbf{1}}+\rho D_{1}^{K, T} \nabla_{T} \nabla^{2} \rho_{1}$ \\
\hline Total species diffusion flux & $J_{1}+\mathcal{J}_{1}$ \\
\hline $\begin{array}{l}\text { Fickian, barodiffusion } \\
\text { and Soret species diffusion flux }\end{array}$ & $\boldsymbol{J}_{\mathbf{1}}=-\rho D_{1,1}^{M} \nabla X_{1}-\rho D_{1}^{P} \nabla P-\rho D_{1}^{T} \nabla T$ \\
\hline Interfacial species diffusion flux & $\mathcal{J}_{1}=\rho D_{1}^{K, M} \nabla_{T} \nabla^{2} \rho_{1}$ \\
\hline
\end{tabular}
(35), $a_{i N}=W_{i} W_{N} / W^{2}$ and $D_{i N}=W^{2} \mathrm{R}^{0} L_{i i} /\left(c W_{i}^{2} W_{N}^{2} X_{i} X_{N}\right)$ are prefactors, and $k_{i T}=W_{i} W_{N} X_{i} X_{N} L_{i q}$ $/\left(W \mathrm{R}^{0} L_{i i}\right)$ is the thermal-diffusion ratio.

Table 1. Transport coefficients and fluxes for a binary mixture with $\kappa_{1,2}=\kappa_{2,1}=\kappa_{2,2}=0$.

Simplified versions of the expressions given above are provided in Table 1 for binary mixtures in which $\kappa_{1,1}$ is assumed to be the only non-zero gradient-energy coefficient for the reasons described in Section III.A after Eq. (3). Additionally, Table 1 lists the coefficients $D_{1}^{K, M}$ and $D_{1}^{K, T}$, which are proportional to the gradientenergy coefficient $\kappa_{1,1}$ and are related to species and heat transport across interfaces. The formulation provided here can be easily simplified to the single-component gradient theory of Ref., ${ }^{17}$ which provides the spatial distribution of density across a stationary quasi-planar vapor-liquid interface. The reduction is based on imposing the mechanical equilibrium condition $-\nabla P_{\mathrm{NL}}+\nabla \cdot \mathcal{K}=0$ in the momentum conservation 
equation (6), with $P_{\mathrm{NL}}$ and $\mathcal{K}$ being defined in Eqs. (9) and (23), respectively. This constraint leads to the gradient-theory equation

$$
P-P_{0}=\kappa \rho \frac{d^{2} \rho}{d \xi^{2}}-\frac{1}{2} \kappa\left(\frac{d \rho}{d \xi}\right)^{2},
$$

where $\xi$ is the coordinate normal to the interface and $P_{0}$ the thermodynamic pressure far from the interface. Integration of Eq. (36), subject to far-field boundary conditions for the vapor- and liquid-phase densities, yields $\rho(\xi)$ across the interface. Further details on the integration of Eq. (36) can be found, for instance, in Ref. $^{34}$

\section{Conclusions}

This study focuses on theoretical aspects of transcritical dynamics of liquid-fuel streams injected into high-pressure environments. The mixture displays a critical-point elevation property by which the twophase region extends up to pressures much larger than the critical pressures of the individual components. As a result, linear thermodynamic trajectories emulating typical injection conditions frequently pass through the two-phase region, thus indicating that the mixture could become separated into liquid and vapor phases by an interface. A set of modifications to the Navier-Stokes equations for multi-component flows is proposed based on diffuse-interface theory in order to treat the emergent and vanishing interfaces in the same flow field. This requires appropriate alterations of the stress tensor and diffusive fluxes of heat and species. The resulting transport formulation is particularized for binary mixtures and single-component flows, the latter recovering the well-known gradient theory of van der Waals. ${ }^{17}$

It should be mentioned that in most practical cases the computational cost of the numerical resolution of the resulting interfaces would be prohibitive, since they typically remain small with respect to the hydrodynamic scales (e.g., see resulting thicknesses based on the one-dimensional gradient theory in Ref. ${ }^{20}$ ). Nonetheless, this should not deter the derivation and understanding of formulations that may be later used to inspire subgrid-scale interface-modeling approaches. Future work will involve the integration of these equations in simple canonical problems.

\section{Acknowledgments}

This investigation was funded by the US AFOSR, Grant \#FA9550-15-C-0037.

\section{References}

\footnotetext{
${ }^{1}$ Oefelein, J. C. \& Yang, V. 1993 Comprehensive review of liquid-propellant combustion instabilities in F-1 engines. J. Propul. Power 9, 657-677.

${ }^{2}$ Rosner, D. E. 1972 Liquid droplet vaporization and combustion. In Liquid Propellant Rocket Combustion Instability, Harrje, D. T. and Reardon H. (Eds.) NASA.

${ }^{3}$ Sirignano, W. A. \& Delplanque, J. P. 1999 Transcritical vaporization of liquid fuels and propellants. J. Propul. Power 15, $806-902$.

${ }^{4}$ Dahms, R. N., Manin, J., Pickett, L. M. \& Oefelein, J. C. 2013 Understanding high-pressure gas-liquid interface phenomena in diesel engines. Proc. Combust. Inst. 34, 1667-1675.

${ }^{5}$ Lasheras, J. C \& Hopfinger, E. J. 2000 Liquid jet instability and atomization. Annu. Rev. Fluid Mech. 32, $275-308$.

${ }^{6}$ Sánchez, A. L., Urzay, J. \& Liñán, A. 2015 The role of separation of scales in the description of spray combustion. Proc. Combust. Inst. 35, 1549-1577.

${ }^{7}$ Gaillard, P., Giovangigli, V. \& Matuszewski, L. 2016 A diffuse interface LOX/hydrogen transcritical flame model. Combust. Theor. Model. 20, 486-520.

${ }^{8}$ Manin, J., Bardi, M., Pickett, L. M., Dahms, R. N. \& Oefelein, J. C. 2014 Microscopic investigation of the atomization and mixing processes of diesel sprays injected into high pressure and temperature environments. Fuel 134, 531-543.

${ }^{9}$ Qiu, L. \& Reitz, R. D. 2015 An investigation of thermodynamic states during high-pressure fuel injection using equilibrium thermodynamics. Int. J. Multiphase Flow 72, 24-38.

${ }^{10}$ Firoozabadi, A. 2015 Thermodynamics and Applications in Hydrocarbon Energy Production. McGraw-Hill.

${ }^{11}$ Heidemann, R. A. \& Khalil, A. M. 1980 The calculation of critical points. AIChE J. 26, 769-779.

${ }^{12}$ Peng, D.-Y. \& Robinson, D. B. 1976 A new two-constant equation of state. Ind. Eng. Chem. Fundam. 15, 59-64.

${ }^{13}$ Harstad, K. G., Miller, R. S. \& Bellan, J. 1997 Efficient high-pressure state equations. AIChE J. 43, 1605-1610.

${ }^{14}$ García-Córdova, T., Justo-García, D. N., García-Flores, B. E. \& García-Sánchez, F. 2011 Vapor-liquid equilibrium data for the Nitrogen + Dodecane system at temperatures from (344 to 593) K and at pressures up to $60 \mathrm{MPa}$. J. Chem. Eng. Data 56, $1555-1564$.
} 
${ }^{15}$ van Konynenburg, P. H. \& Scott, R. L. 1980 Critical lines and phase equilibria in binary van der Waals mixtures. Philos. Trans. Royal Soc. Lond. A 298, 495-540.

${ }^{16}$ Matheis, J. \& Hickel S. 2016 Multi-component vapor-liquid equilibrium model for LES and application to ECN Spray A. Proceedings of the Summer Program, Center for Turbulence Research, Stanford University, pp. 25-34.

${ }^{17}$ van der Waals, J. D. 1893 The thermodynamic theory of capillarity under the hypothesis of a continuous variation of density. Translated by J. S. Rowlinson 1979 J. Stat. Phys. 20, 197-244.

${ }^{18}$ Cahn, J. W. \& Hilliard, J. E. 1958 Free energy of a nonuniform system. I. Interfacial free energy. J. Chem. Phys. 28, $258-267$.

${ }^{19}$ Anderson, D. M., McFadden, G. B. \& Wheeler, A. A. 1998 Diffuse-interface methods in fluid mechanics. Annu. Rev. Fluid Mech. 30, 139-165.

${ }^{20}$ Dahms, R. N. \& Oefelein, J. C. 2013 On the transition between two-phase and single-phase interface dynamics in multicomponent fluids at supercritical pressures. Phys. Fluids 25, 092103.

${ }^{21}$ Dunn, J. E. \& Serrin, J. 1985 On the thermomechanics of interstitial working. Arch. Ration. Mech. Anal. 88, 95-133.

${ }^{22}$ Pismen, L. M. 2001 Nonlocal diffuse interface theory of thin films and the moving contact line. Phys. Rev. E 64, 021603.

${ }^{23}$ Rowlinson, J. S. \& Widow, B. 2002 Molecular Theory of Capillarity. Dover.

${ }^{24}$ Lin, H., Duan, Y.-Y. \& Min, Q. 2007 Gradient theory modeling of surface tension for pure fluids and binary mixtures. Fluid Phase Equilib. 254, 75-90.

${ }^{25}$ Benedict, M., Webb, G. B. \& Rubin, L. C. 1942 An empirical equation for thermodynamic properties of light hydrocarbons and their mixtures II. Mixtures of methane, ethane, propane, and n-butane. J. Chem. Phys. 10, 747-758.

${ }^{26}$ Soave, G. 1972 Equilibrium constants from a modified Redlich-Kwong equation of state. Chem. Eng. Sci. 27, $1197-1203$.

${ }^{27}$ Chung, T. H., Ajlan, M., Lee, L. L. \& Starling, K. E. 1988 Generalized multiparameter correlation for nonpolar and polar fluid transport properties. Ind. Eng. Chem. Fundam. 27, 671-679.

${ }^{28}$ Poling, B. E., Prausnitz, J. M. \& O'Connel, J. P. 2001 The Properties of Gases and Liquids. McGraw-Hill.

${ }^{29}$ Miller, R. S. 2000 Long time mass fraction statistics in stationary compressible isotropic turbulence at supercritical pressure. Phys. Fluids 12, 2020-2032.

${ }^{30}$ Papatzacos, P. 2000 Diffuse-interface models for two-phase flow. Physica Scripta 61, 349-360.

${ }^{31}$ Landau, L. D. \& Lifshitz, E. M. 1987 Course of Theoretical Physics: Fluid mechanics. Pergamon Press.

${ }^{32}$ Onsager, L. 1931 Reciprocal relations in irreversible processes. I. Phys. Rev. 37, 405-426.

${ }^{33}$ Curie, P. 1894 Sur la symétrie des phénomènes physiques: symétrie d'un champ électrique et d'un champ magnétique. $J$. Phys. 3, 393-415.

${ }^{34}$ Jofre, L., Urzay, J., Mani, A. \& Moin, P. 2015 On diffuse-interface modeling of high-pressure transcritical fuel sprays. Annual Research Briefs, Center for Turbulence Research, Stanford University, 55-64. 